\title{
Contribuição da Revista Ciência \& Saúde Coletiva para a área de Alimentação e Nutrição no Brasil
}

\author{
Contribution to the Journal Ciência \& Saúde Coletiva \\ on Food and Nutrition in Brazil
}

\author{
Vania Matos Fonseca (http://orcid.org/0000-0002-5452-7081) ${ }^{1}$ \\ Fernanda Rebelo (http://orcid.org/0000-0001-6207-5161) ${ }^{1}$ \\ Daniele Marano (http://orcid.org/0000-0001-6985-941X) ${ }^{1}$ \\ Andrea Dunshee de Abranches (https://orcid.org/0000-0002-9323-3297) ${ }^{1}$ \\ Yasmin Notarbartolo di Villarosa do Amaral (http://orcid.org/0000-0001-8159-0564) ${ }^{2}$ \\ Vanessa Mendes Xavier (https://orcid.org/0000-0001-6586-9469) ${ }^{1}$ \\ Francisco de Assis Guedes de Vasconcelos (http://orcid.org/0000-0002-6162-8067) ${ }^{3}$
}

${ }^{1}$ Unidade de Pesquisa Clínica, Instituto Nacional de Saúde da Mulher, da Criança e do Adolescente Fernandes Figueira (IFF), Fundação Oswaldo Cruz (Fiocruz). Av. Rui Barbosa 716, Flamengo. 22250-020 Rio de Janeiro RJ Brasil. vaniamf36@hotmail.com 2 Programa de PósGraduação em Pesquisa Aplicada à Saúde da Criança e da Mulher, IFF, Fiocruz. Rio de Janeiro RJ Brasil. ${ }^{3}$ Programa de PósGraduação em Nutrição, Universidade Federal de Santa Catarina. Florianópolis SC Brasil.
Abstract A systematic review conducted in January 2020 using SciELO database with the objective of analyzing the scientific production from 19962019, of the Journal Ciência \& Saúde Coletiva in the area of food and nutrition. We selected 509 out of the 904 articles screened by titles and abstracts. We grouped the articles into ten themes and discussed the most frequent ones: Nutritional Status Assessment ( $n=142)$, Food Intake $(n=111)$, Food and Nutrition Policies and Programmes $(n=105)$ and Breastfeeding $(n=35)$. The publications were mostly original articles (75.6\%) employing quantitative method (81.6\%) and, among these, $18.8 \%$ used a probabilistic sampling. We assembled a wide range of topics and subthemes, a relevant production and repository of data and knowledge for health professionals and managers. As gaps, there was a scarcity of publications focused on micronutrient deficiency; the promotion of the Food Guide for the Brazilian Population; supporting the rise of breastfeeding, the impact and analyses of the disruption of the National Food and Nutrition Security Policy and its multi sector interactions with social policies to fight hunger. Key words Food and Nutrition, Scientific Production, Literature Review, Journal Ciência \& Saúde Coletiva
Resumo Revisão sistemática realizada em janeiro de 2020 na base de dados SciELO com o objetivo de analisar a produção científica da Revista Ciência \& Saúde Coletiva na área de alimentação e nutrição no período 1996-2019. A busca resultou em 904 artigos e 509 foram selecionados após leitura dos títulos e resumos. Os artigos foram agrupados em dez temas, sendo discutidos os de maior frequência: Avaliação do Estado Nutricional ( $n=142)$, Consumo Alimentar ( $n=111)$, Politicas e Programas de Alimentação e Nutrição $(n=105)$ e Aleitamento Materno $(n=35)$. As publicações foram em sua maioria artigos originais $(75,6 \%)$ com método quantitativo $(81,6 \%)$ e, entre estes, $18,8 \%$ utilizaram amostra probabilística. Observou-se um amplo leque de temas e subtemas abordados, evidenciando uma produção relevante que constitui um repositório importante de dados e conhecimentos para profissionais e gestores da área da saúde. Como lacunas, observou-se a escassez de publicações voltadas para a deficiência de micronutrientes; a popularização do Guia Alimentar para População Brasileira; a promoção da ascensão do aleitamento materno; os diagnósticos da descontinuidade da Política Nacional de Segurança Alimentar e Nutricional e suas articulações intersetoriais com as políticas sociais de combate à fome.

Palavras-chave Alimentação e Nutrição, Produção Científica, Revisão de Literatura, Revista Ciência \& Saúde Coletiva 


\section{Introdução}

A nutrição como ciência, política pública e profissão teve sua emergência no Brasil nos primeiros anos da década de 1930. Caracteriza-se como um campo complexo e multidisciplinar, embora a formação profissional tenha sido localizada historicamente no setor saúde e centralizada em enfoques das ciências biológicas ${ }^{1,2}$.

Nas últimas três décadas, a sociedade brasileira se defrontou com profundas alterações econômicas, políticas e sociais, as quais também se refletiram no campo da nutrição. Entre estas podem ser destacadas as relacionadas ao cenário epidemiológico nutricional que passou a configurar-se no país a partir da década de 1990. Cenário caracterizado, por um lado, pela manutenção de doenças nutricionais relacionadas à miséria, à pobreza e ao atraso econômico (desnutrição energético protéica, deficiência de vitamina A, anemia ferropriva, etc.), mais prevalentes no contexto histórico de emergência deste campo científico no país, os anos 1930. Por outro lado, a ascensão de doenças nutricionais associadas à riqueza, ao avanço tecnológico e à modernidade (obesidade, diabetes, dislipidemias, hipertensão, certos tipos de câncer, etc.), mais prevalentes no contexto atual $^{3-7}$.

Faz sentido, portanto, que o primeiro artigo da área de nutrição publicado pela Revista Ciência \& Saúde Coletiva (C\&SC), em seu volume 7, número 2, do ano de 2002, tenha sido na modalidade de debate e intitulado "para a crítica da transição nutricional", um conceito emergente no país em meados da década de 1990 e que sinalizava a necessidade de adoção de novos paradigmas explicativos e intervencionistas para a nova realidade alimentar e nutricional brasileira ${ }^{2,5}$.

Alguns estudos têm investigado a produção científica do campo da nutrição, publicada em periódicos nacionais, a partir de distintos objetivos, enfoques conceituais e procedimentos metodológicos ${ }^{9,10}$. Olinto et al. ${ }^{9}$ mostraram que na lista dos 20 periódicos com maior número de publicações pelos Programas de Pós-graduação em Nutrição, avaliados no período de 2007 a 2009, os três com maiores volumes foram: Revista de Nutrição $(\mathrm{n}=100)$, Cadernos de Saúde Pública $(\mathrm{n}=63)$ e C\&SC $(\mathrm{n}=63)^{9}$. Vasconcelos ${ }^{10}$, por sua vez, em revisão da produção científica do campo da nutrição publicada pela Scientific Electronic Library (SciELO) até o mês de setembro de 2016, observou que dos 779 artigos publicados em 85 periódicos, os 5 periódicos com maiores volumes foram: Revista de Nutrição $(\mathrm{n}=177)$, Cadernos de Saúde Pública ( $\mathrm{n}=84)$, Revista de Saúde Pública $(n=60)$, C\&SC $(n=50)$ e Jornal de Pediatria $(\mathrm{n}=36)^{10}$.

Este artigo tem por objetivo analisar a produção científica da C\&SC na área de Alimentação e Nutrição (A\&N) ao longo do período 1996 a 2019, procurando realizar breve diálogo com a literatura científica no cenário internacional.

\section{Métodos}

Trata-se de revisão sistemática dos artigos publicados na C\&SC, na área de A\&N, desde a sua criação, em 1996, até o ano de 2019, inclusive.

A busca foi realizada na base de dados SciELO no dia 7 de janeiro de 2020, utilizando-se o filtro da C\&SC. Para a escolha das palavras-chave foi realizado um brainstorm entre os autores, visando reunir todos os termos relacionados à área de A\&N. A partir desses termos os índices de assunto do SciELO foram consultados para capturar todas as palavras com radicais semelhantes e seus diversos sufixos, em português, espanhol e inglês.

Essa estratégia de busca resultou nas seguintes palavras-chave, que foram pesquisadas de forma a incluir publicações que as contivessem no título ou resumo: "NUTRICAO or NUTRICIO or NUTRICIONAIS or NUTRICIONAL or NUTRICIONISTA or NUTRIENTES or NUTRIENTS or NUTRITION or NUTRITIONAL or NUTRITIONIST or NUTRITIVE or NUTRITIVOS or NUTRIZ OR MICRONUTRIENT or MICRONUTRIENTE or MICRONUTRIENTS or MICRONUTRIENTES or ALIMENTA or ALIMENTACAO or ALIMENTACION or ALIMENTADA or ALIMENTAM or ALIMENTAN or ALIMENTANDO or ALIMENTAR or ALIMENTARAM or ALIMENTAREM or ALIMENTARES or ALIMENTARIA or ALIMENTARIO or ALIMENTARIOS or ALIMENTARIUS or ALIMENTARY or ALIMENTICIOS or ALIMENTO or ALIMENTOS or ALIMENTOSA or DIET or DIETA or DIETARIA or DIETARY or DIETARYRISK or DIETAS or DIETETIC or DIETETICA or DIETETICAS or DIETETICO or DIETETICOS or DIETETICS or INGESTA or INGESTAO or INGESTION or FEED or FEEDING or CONSUMO or CONSUMPTION or FOOD or FOODBORNE or FOODCONTROL or FOODS or FOODSERVICE or OBESIDAD or OBESIDADE or OBESITY or SOBREPESO or OVERWEIGHT or DESNUTRICAO or UNDERNUTRITION or ANTROPOMETRIA or ANTHROPOMETRIC or ANTHROPOMETRY or AMAMENTACAO or AMAMENTADAS 
or AMAMENTADOS or AMAMENTANDO or AMAMENTAR or AMAMENTARAM or AMAMENTOU or BREASTFED or BREASTFEED or BREASTFEEDING".

A busca retornou um total de 904 publicações. A partir da leitura de títulos e resumos foram excluídas 395 publicações que não se enquadravam na área de A\&N, resultando em 509 artigos incluídos.

As publicações selecionadas foram avaliadas em relação as seguintes informações: ano de publicação, temas, palavras-chave, tipo de artigo (artigo original, revisão narrativa, revisão sistemática, editorial, opinião, resenha, carta), tipo de estudo (quantitativo, qualitativo ou quali-quantitativo), grupo populacional estudado (neonatos, lactentes, escolares, adolescentes, mulheres adultas, homens adultos, adultos de ambos os sexos, idosos, gestantes, lactantes, puérperas e grupo materno-infantil) grupos minoritários (indígenas, quilombolas, população rural/assentamentos e refugiados), amostra probabilística ( $\operatorname{sim}$ ou não) e país de realização.

As publicações foram agrupadas em dez temas: Avaliação do Estado Nutricional (A), Consumo Alimentar (B), Políticas e Programas de A\&N (C), Amamentação e Alimentação Complementar (D), Nutrição Comportamental (E), Educação Nutricional (F), Cuidado nutricional dos grupos populacionais (G), Profissão do Nutricionista (H), Higiene dos Alimentos (I) e Tecnologia de Alimentos ( $\mathrm{J}$ ). Os resultados foram apresentados na forma de tabelas e gráficos e discutidos de acordo com o contexto histórico, social e cultural, além de comparados com a literatura internacional. Visto a magnitude e diversidade da área, optou-se por direcionar a discussão dos resultados para os temas $\mathrm{A}, \mathrm{B}, \mathrm{C}$ e D que concentraram o maior número de publicações.

Com o objetivo de realizar breve diálogo entre a produção científica da C\&SC na área de $\mathrm{A} \& \mathrm{~N}$ com a literatura científica no cenário internacional, realizou-se busca bibliográfica na base PubMed (U.S. National Library of Medicine/U.S. National Institutes of Health). Esta busca teve como recorte temporal o período de 1996 a 2019 inclusive e utilizou-se unitermos específicos para os quatro temas mais frequentes já especificados $\operatorname{acima}(\mathrm{A}, \mathrm{B}, \mathrm{C}$ e $\mathrm{D})$.

\section{Resultados}

\section{Características bibliométricas gerais dos artigos da área de $A \& N$}

Considerando o total de 4414 publicações da C\&SC no período avaliado, os 509 artigos na área de $\mathrm{A} \& \mathrm{~N}$ representam $11,5 \%$. Os temas mais abordados na revista foram: Avaliação do Estado Nutricional (27,9\%), Consumo Alimentar (21,8\%), Políticas e Programas de A\&N (20,6\%), Amamentação e Alimentação Complementar $(6,9 \%)$. Dentro desses temas, os principais grupos de publicaçõesabordados foram avaliação do estado nutricional e seus determinantes $(26,7 \%)$, consumo de alimentos ou grupos alimentares $(16,1 \%)$ e segurança alimentar e nutricional (14,5\%) (Tabela 1).

Três quartos das publicações da área $(75,6 \%)$ são artigos originais. Desses, $81,6 \%$ são quantitativos. Entre os estudos quantitativos, 18,8\% utilizaram amostras probabilísticas. Os artigos apresentam uma distribuição equilibrada entre os grupos populacionais, sendo $28,5 \%$ voltados aos adultos e $21,8 \%$ direcionado às crianças, de lactentes a escolares. Poucas publicações são voltadas a grupos minoritários $(5,1 \%)$ e $95,1 \%$ foi realizado no Brasil (Tabela 2 ).

A primeira publicação na C\&SC relacionada ao tema $A \& N$ ocorreu em 2002, seis anos após a criação do periódico. A partir de então as publicações na área se mantiveram na média de 3,5 artigos/ano até 2009. Desde 2010 as publicações multiplicaram, mantendo uma média de 47,7 artigos/ano até 2019. Considerando que até 2006 a revista tinha periodicidade trimestral, de 2007 a 2010 se tornou bimestral e em 2011 passou a ter a periodicidade mensal, o aumento superou o esperado, já que a periodicidade aumentou em média 3 vezes, enquanto a média de publicações/ ano multiplicou em 13 vezes. Os anos com maior quantitativo de publicações foram $2014(\mathrm{n}=61)$ e $2019(n=63)$ (Figura 1).

\section{Discussão}

\section{Os artigos publicados sobre o tema avaliação do estado nutricional}

O tema avaliação do estado nutricional foi dividido em dois grupos de publicações: avaliação do estado nutricional e seus determinantes $(95,8 \%)$ e métodos de avaliação nutricional $(4,2 \%)$ (Tabela 1). 


\begin{tabular}{|c|c|c|c|c|}
\hline \multirow[t]{26}{*}{ 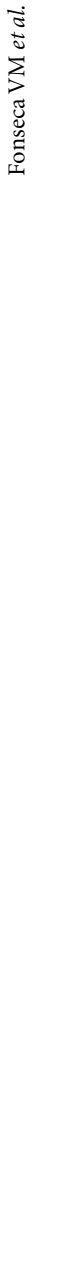 } & $\begin{array}{l}\text { Tabela 1. Frequência de artigos publicados na área de } \\
\text { Coletiva de acordo com os temas, 1996-2019. }\end{array}$ & Nut & Revista Ciên & \& Saúde \\
\hline & Temas & $\mathbf{n}$ & $\%$ do total & $\%$ do tema \\
\hline & A. Avaliação do estado nutricional & 142 & 27,9 & \\
\hline & Avaliação do estado nutricional e seus determinantes & 136 & 26,7 & 95,8 \\
\hline & Métodos de avaliação nutricional & 6 & 1,2 & 4,2 \\
\hline & B. Consumo alimentar & 111 & 21,8 & \\
\hline & Consumo de nutrientes/micronutrientes & 16 & 3,1 & 15,8 \\
\hline & Consumo de alimentos/grupos alimentares & 82 & 16,1 & 81,2 \\
\hline & Consumo de ultraprocessados & 3 & 0,5 & 3,0 \\
\hline & Métodos de avaliação de consumo & 10 & 2,0 & 62,5 \\
\hline & C. Políticas e programas de alimentação e nutrição & 105 & 20,6 & \\
\hline & Segurança alimentar e nutricional & 74 & 14,5 & 70,5 \\
\hline & Alimentação escolar & 26 & 5,1 & 24,8 \\
\hline & Saúde do trabalhador & 5 & 0,9 & 4,8 \\
\hline & D. Amamentação e alimentação complementar & 35 & 6,9 & \\
\hline & Aleitamento materno & 28 & 5,5 & 80,0 \\
\hline & Introdução alimentar/alimentação complementar & 7 & 1,4 & 20,0 \\
\hline & E. Nutrição comportamental & 35 & 6,9 & \\
\hline & F. Educação nutricional & 23 & 4,5 & \\
\hline & G. Cuidado nutricional dos grupos populacionais & 20 & 3,9 & \\
\hline & H. Profissão do nutricionista & 19 & 3,7 & \\
\hline & Atuação profissional do nutricionista & 10 & 2,0 & 52,6 \\
\hline & História da nutrição & 9 & 1,7 & 47,4 \\
\hline & I. Higiene dos alimentos & 13 & 2,6 & \\
\hline & J. Tecnologia de alimentos & 6 & 1,2 & \\
\hline & Total & 509 & 100 & \\
\hline
\end{tabular}

Os estudos sobre métodos de avaliação nutricional, resumidamente, buscaram avaliar a validade e precisão de diferentes métodos na avaliação nutricional de grupos populacionais diversos. Sobre avaliação do estado nutricional e seus determinantes, as publicações na C\&SC tiveram início em 2007 e representaram 26,7\% $(n=136)$ do montante de artigos da área de A\&N (Tabela 1). Esse quantitativo é bastante expressivo e contribuiu, em conjunto com outras revistas, para o diagnóstico nutricional da população brasileira.

Estudos nessa temática são especialmente importantes para a vigilância nutricional, direcionando profissionais de saúde e gestores. A vigilância norteia a modificação da agenda de $A \& N$ do Sistema Único de Saúde (SUS) e culmina com publicações que visam apresentar diretrizes oficiais para a promoção da alimentação adequada e saudável, tais como: Dez passos para uma alimentação saudável ${ }^{11}$; Novo guia alimentar para população brasileira ${ }^{12}$; e o Guia alimentar para menores de $2 \operatorname{anos}^{13}$.
As investigações nesse tema apresentaram como principal foco a obesidade, o sobrepeso e/ ou excesso de peso, representando $47 \%$ da produção do tema. Por outro lado, artigos focados no estudo da desnutrição protéico-calórica e baixo peso somaram apenas $10 \%$. Esse perfil de publicações reflete a transição nutricional no Brasil, com declínio da prevalência de baixo peso e aumento da obesidade em todas as regiões, independentemente de sexo, idade e fatores socioeconômicos ${ }^{5,8,14}$. De acordo com dados da Pesquisa Nacional de Saúde, o excesso de peso foi verificado em $56,9 \%$ da população brasileira adulta em 2013, enquanto que a mesma pesquisa mostrou prevalência de déficit de peso de apenas $2,5 \%$ para o mesmo grupo ${ }^{15}$. Ao redor do mundo, o número de obesos dobrou em 35 anos e pessoas com excesso de peso já representam um terço da população mundial ${ }^{16}$.

Nos artigos publicados na C\&SC, a obesidade foi estudada pelos mais diversos pontos de vista. Algumas publicações exploraram o aspecto social 
Tabela 2. Características bibliométricas gerais dos artigos da área de alimentação e nutrição publicados na Revista Ciência \& Saúde Coletiva, 1996-2019.

\begin{tabular}{|c|c|c|}
\hline & $\mathbf{n}$ & $\%$ \\
\hline \multicolumn{3}{|l|}{ Tipo de artigo } \\
\hline Artigo original & 385 & 75,6 \\
\hline Revisão narrativa & 47 & 9,2 \\
\hline Revisão sistemática & 50 & 9,8 \\
\hline Editorial & 9 & 1,8 \\
\hline Opinião & 9 & 1,8 \\
\hline Resenha & 2 & 0,4 \\
\hline Carta & 3 & 0,6 \\
\hline Debate & 1 & 0,2 \\
\hline Press release & 3 & 0,6 \\
\hline \multicolumn{3}{|l|}{ Tipo de estudo ${ }^{\alpha}$} \\
\hline Quantitativo & 314 & 81,6 \\
\hline Qualitativo & 64 & 16,6 \\
\hline Quali-quantitativo & 7 & 1,8 \\
\hline \multicolumn{3}{|l|}{ Amostra probabilística ${ }^{\beta}$} \\
\hline Sim & 59 & 18,8 \\
\hline Não & 255 & 81,2 \\
\hline \multicolumn{3}{|c|}{ Grupo populacional/Faixa etária } \\
\hline Crianças & 111 & 21,8 \\
\hline Adolescentes & 72 & 14,1 \\
\hline Adultos & 145 & 28,5 \\
\hline Idosos & 52 & 10,2 \\
\hline Não delimitado & 53 & 10,4 \\
\hline Não se aplica & 92 & 18,1 \\
\hline \multicolumn{3}{|l|}{ Grupo minoritário } \\
\hline Sim & 26 & 5,1 \\
\hline Não & 483 & 94,9 \\
\hline \multicolumn{3}{|l|}{ Realizado no Brasil } \\
\hline Sim & 484 & 95,1 \\
\hline Não & 25 & 4,9 \\
\hline
\end{tabular}

e cultural, evidenciando como as desigualdades influenciam no acesso aos meios de prevenção e combate à obesidade, principalmente entre as mulheres. Outros, fizeram um panorama da obesidade no Brasil, com dados de base populacional e muitas vezes observando o aspecto temporal, da variação deste agravo ao longo dos anos, em diferentes grupos populacionais. Adicionalmente, foi encontrada variedade de artigos buscando elucidar os fatores associados ao excesso de peso, avaliando principalmente variáveis de estilo de vida e socioeconômicas, mas também peso ao nascer, disruptores endócrinos presentes na água e transmissão intergeracional.
A busca no PubMed utilizando a palavrachave nutritional status resultou em 5.686 artigos no período de interesse, mostrando ser um tema frequente na literatura internacional, assim como na C\&SC foi o tema de maior ocorrência. Quando se pesquisou pelas palavras obesity e malnutrition foram encontrados 124.028 e 41.436 resultados, respectivamente. Dessa forma, observamos que o maior interesse para o problema da obesidade em detrimento da desnutrição nos últimos 25 anos é uma tendência internacional.

É importante frisar que as deficiências de micronutrientes, em especial anemia ferropriva em crianças e mulheres em idade fértil, ainda é motivo de preocupação no Brasil ${ }^{14}$. Estima-se que mais de $40 \%$ das crianças brasileiras apresentem anemia, podendo chegar a prevalências maiores que $70 \%$ em menores de dois anos, sendo considerado um grave problema de saúde pública ${ }^{17}$. Apenas 6\% dos artigos publicados no tema de avaliação do estado nutricional e seus determinantes foram voltados para as deficiências de micronutrientes, sendo metade destes com crianças e/ou adolescentes e somente um com gestantes, o que indica que o tema merece maior atenção por parte da C\&SC.

Aproximadamente um terço dos estudos publicados nessa temática não teve como cerne um agravo nutricional específico, mas sim descreveram a avaliação nutricional e seus múltiplos resultados, sendo estes oriundos majoritariamente de medidas antropométricas. Entre os principais grupos populacionais estudados estão portadores de doenças (demência, doença renal crônica, hanseníase, fibrose cística e câncer de mama), crianças, adolescentes, mulheres e idosos. Observou-se também alguns estudos direcionados a grupos minoritários, como quilombolas, indígenas, beneficiários do bolsa família e população rural.

\section{Os artigos publicados sobre o tema consumo alimentar}

As publicações sobre consumo alimentar tiveram início em 2003 e durante esses 16 anos a C\&SC publicou 16 artigos sobre consumo de nutrientes e micronutrientes, 82 sobre consumo de alimentos/grupo de alimentos e apenas 3 artigos sobre alimentos ultraprocessados.

Os artigos que avaliaram o consumo de nutrientes e micronutrientes se detiveram a grupos populacionais específicos. Em crianças, os estudos avaliaram a associação entre o crescimento linear e a ingestão de ferro e vitamina $\mathrm{A}$, melho- 


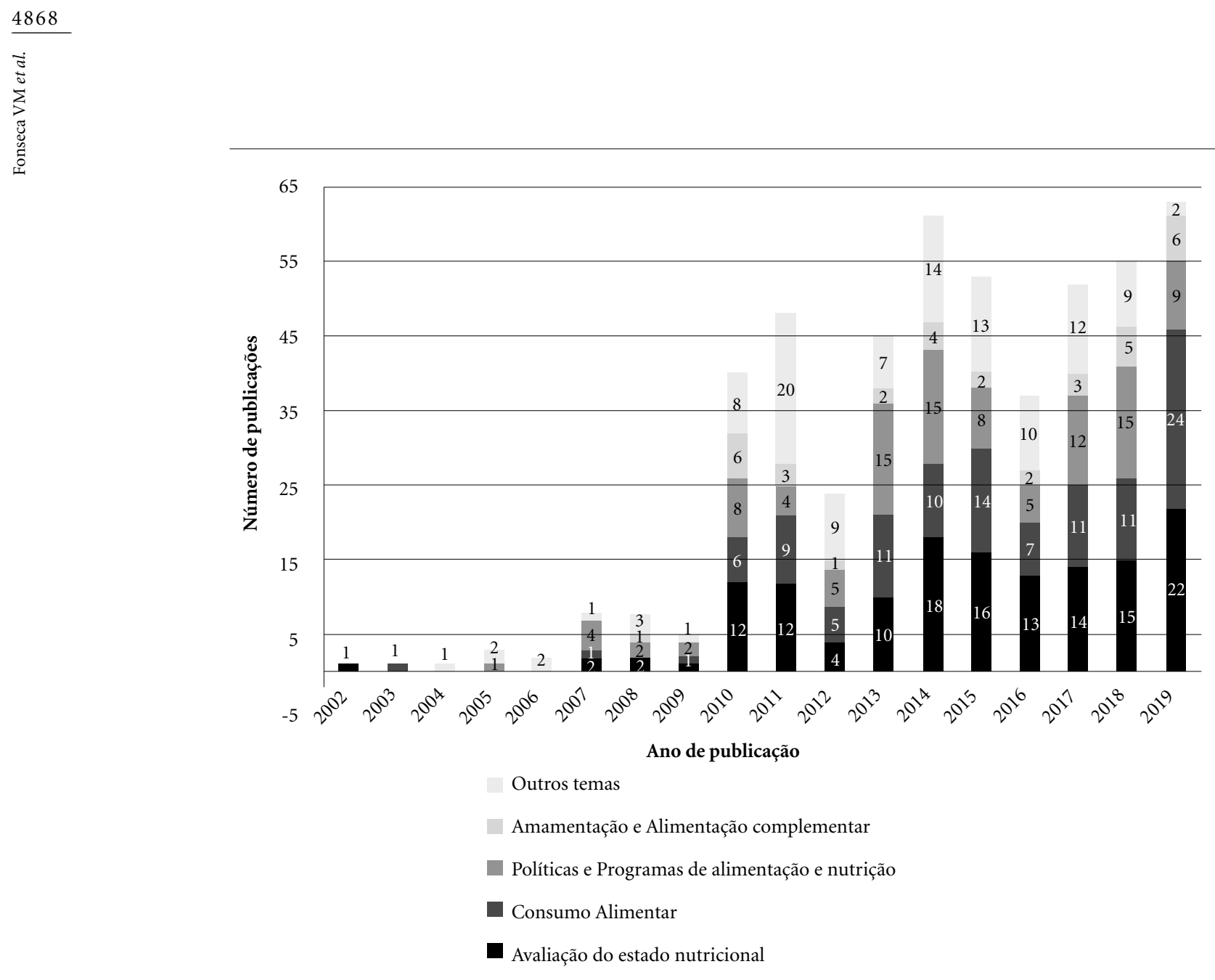

Figura 1. Distribuição anual dos artigos publicados na área de Alimentação e Nutrição na Revista Ciência \& Saúde Coletiva de acordo com os grandes temas, 2002-2019.

ria do estado nutricional de pré-escolares suplementados com micronutrientes e o impacto da suplementação de vitamina A no leite materno. Em adolescentes, os artigos avaliaram a associação entre o excesso de peso corporal e o consumo inadequado de nutrientes e alimentos em escolas públicas e apontaram risco de deficiência de ferro e cálcio. Em adultos, as discussões se concentraram na avaliação das dietas ricas em gorduras e risco de doenças cardiovasculares, nas intervenções utilizando educação nutricional e atividade física.

Os artigos sobre consumo de alimentos/grupos alimentares se detiveram na avaliação dos hábitos alimentares, disponibilidade de alimentos, consumo de álcool, alimentação saudável e qualidade da dieta, publicidade de alimentos e alimentos de rua em grupos populacionais específicos (gestantes, idosos, adolescentes, população rural, indígenas, trabalhadores e crianças).
Observou-se que houve um crescimento expressivo no quantitativo de publicações dessas temáticas nas últimas décadas. Minayo e Gualhano ${ }^{18}$ apontaram a qualidade da produção e consumo de alimentos como um dos maiores desafios da saúde pública contemporânea.

Quando avaliado o consumo alimentar em relação às variáveis sociodemográficas e econômicas, a análise dos dados da Pesquisa Nacional de Demografia e Saúde (PNDS) apontou maior consumo de dieta saudável em crianças entre 2 e 5 anos de mães com maior nível educacional ${ }^{19}$. Em adolescentes, os resultados da Pesquisa Nacional de Saúde do Escolar mostraram relação direta entre o consumo de refrigerantes e o nível socioeconômico. Já o consumo de feijão na última grande refeição $(62,6 \%)$ esteve presente em todos os grupos socioeconômicos ${ }^{20}$. Alguns artigos apontaram que feijão é um marcador da dieta dos brasileiros e sua mistura com o arroz 
foi associada a hábitos saudáveis e menor sobrepeso. Em adultos, observou-se maior ingestão de carboidratos entre indivíduos de baixa renda e maior consumo de lipídios entre aqueles com renda elevada ${ }^{21}$. Estudos observaram maior dificuldade em adotar dieta saudável entre idosos, ocasionando deficiências na ingestão de nutrientes $^{22}$. Em grupos vulneráveis, como gestantes, nutrizes e obesas, foi evidenciada a necessidade de revisão das estratégias para estimular dietas adequadas $^{23}$, maior entendimento dos aspectos históricos e socioculturais que interferem nas escolhas alimentares ${ }^{24}$, e a necessidade de realização de estudos que tenham abordagem multidimensional ${ }^{25}$.

$\mathrm{Na}$ discussão com artigos publicados na base PubMed ( $\mathrm{n}=755)$ observou-se que desde a metade da década de 1990 o tema dos micronutrientes, em sua capacidade antioxidante e protetora e seu impacto nas doenças crônico-degenerativas e outras afecções, vem sendo discutido. A partir de 2000 surgiram outros temas, como micronutrientes e integridade genômica, uso de micronutrientes associado aos ácidos graxos poliinsaturados e aos probióticos, e ainda pesquisas sobre suplementação em idosos, crianças, grávidas e mulheres na menopausa. Em 2010 outros temas foram incorporados, como: nutrição genômica e crescimento, prevenção terapêutica e imunidade, prevenção de anomalias congênitas, reparação de DNA e infertilidade. Entre 2011/2012, houve crescimento no volume de revisões sistemáticas sobre uso de suplementação para vários grupos etários e patologias, bem como, fortificação em farinhas, programação epigenética, stress oxidativo, função cerebral e neurodesenvolvimento, interações metabólicas entre micronutrientes $\mathrm{e}$ uso de aplicativos para orientar ingestão.

Observou-se aumento ao longo dos últimos 25 anos das publicações internacionais sobre consumo de alimentos/grupos alimentares. $\mathrm{Na}$ década de 1990 muitos estudos contemplaram a estimação do consumo diário de alimentos em grupos etários e países; discutindo renda, hábitos e tipos de alimentos, nas doenças degenerativas, principalmente câncer. A partir de 1996 aparecem estudos sobre aditivos nos alimentos, pesticidas, flavonóides, fibras e imagem corporal em escolares. No final dessa década, foram avaliados o crescimento econômico e as faixas de renda em relação ao consumo alimentar em grupos populacionais diversos. A partir de 2003 destacaram-se estudos sobre fast-food, qualidade da dieta, transição nutricional, escolaridade materna e seus impactos econômicos e sociais no consumo alimentar. Nos anos seguintes surgiram discussões sobre a associação entre assistir televisão e excesso de peso em crianças e avaliação do padrão de consumo alimentar.

Também foram observadas inúmeras publicações sobre o consumo de açúcar, fast-food, junk food, snacks nas merendas escolares e os padrões de consumo associados à mortalidade por doenças cardiovasculares e câncer. A partir de 2010 destacaram-se os estudos sobre consumo em populações de baixa renda, desigualdades sociais e o Programa Bolsa Família (PBF) no Brasil ${ }^{26}$.

Entre 2000 e 2010, muitas publicações se voltaram para discussões sobre "guidelines", estilo de vida, consumo de frutas e vegetais, consumo de grãos integrais, alimentos obesogênicos e alergias alimentares. A partir de 2015 cresceram os debates sobre intolerâncias e impulsividade alimentar, uso de smartphone para auxiliar o comportamento alimentar, identificação de emoções no consumo, alimentos orgânicos e sustentabilidade, genótipo e análise multidimensional associados ao consumo. Em 2019, com a crise democrática, uma revisão de publicações analisou a relação entre posição socioeconômica e consumo alimentar nos grandes inquéritos nacionais, apontando as desigualdades sociais e o consumo no Brasil ${ }^{27}$.

No contexto mundial, desde 2011 iniciaram-se as publicações sobre os alimentos ultraprocessados, sendo o principal autor desses artigos o pesquisador brasileiro Carlos Augusto Monteiro ${ }^{28,29}$. Tais publicações contemplaram o impacto do consumo deste grupo de alimentos na qualidade da dieta, suas repercussões nas doenças crônicas, e em grupos etários específicos (mais vulneráveis como crianças e adolescentes). A C\&SC contribuiu com apenas 3 artigos sobre essa temática, se detendo na avaliação da introdução precoce desses alimentos em crianças menores de 2 anos, consumo de bebidas açucaradas em pré-escolares e aumento do consumo desses alimentos em famílias de maior poder aquisitivo.

\section{Os artigos sobre o tema Políticas e Programas de A\&N}

As publicações sobre Políticas e Programas de $A \& N(n=105)$ foram divididas em 3 grandes grupos: Segurança Alimentar e Nutricional (SAN), Alimentação Escolar e Saúde do Trabalhador que representam 70,5\%, 24,8\% e 4,8\% da produção no tema, respectivamente (Tabela 1).

Sobre SAN, as publicações tiveram início em 2005 e observou-se tendência de crescimen- 
to no número de publicações entre 2014 e 2017. $\mathrm{Na}$ discussão entre os artigos que avaliaram a SAN na C\&SC $(n=74)$ e os artigos publicados no PubMed ( $\mathrm{n}=404)$, observou-se que as publicações internacionais avaliaram, além das temáticas supracitadas, a associação da SAN com doenças como asma e HIV, amamentação, capital social, mudanças climáticas, suplementação nutricional, entre outras.

Diversos programas governamentais voltados para o combate à fome foram criados ao longo do século XX. Todavia, o conceito de SAN surgiu oficialmente em 1985 com a elaboração da proposta de uma Política Nacional de SAN ${ }^{30}$. Em 2006, foi realizada a publicação da Lei Orgânica de SAN (LOSAN) que criou o Sistema Nacional de $\operatorname{SAN}^{3,4,6,7,30}$.

Em 2005, Stoppelli e Magalhães ${ }^{31}$ publicaram o primeiro artigo que teve como objetivo avaliar as ações preventivas para minimizar os efeitos dos agrotóxicos, tais como: maior fiscalização na comercialização e no uso de produtos químicos, simplificação dos rótulos nas embalagens e maior adequação dos equipamentos de proteção. Apesar desse assunto ter sido escassamente abordado na C\&SC, destaca-se que houve aumento de seu uso nas últimas décadas no Brasil. Ademais, em 2019 foi publicado no Diário Oficial da União a liberação de mais de 52 substâncias pelo atual governo, evidenciando retrocessos em relação ao uso de pesticidas, comprometimento da SAN da população e assumimento da dianteira mundial no uso de agrotóxicos ${ }^{32}$.

A temática intersetorialidade foi abordada entre os artigos publicados na C\&SC, dado que perfaz a política de SAN. Observou-se que ainda existe um grande entrave em relação a essa questão e que são necessários avanços na obtenção de ações articuladas e coordenadas que utilizem os recursos existentes em cada setor de modo mais eficiente, direcionando-os para ações que obedeçam a uma escala de prioridades estabelecidas em conjunto para o alcance de uma Política de SAN efetiva $^{3,4,6,7,30}$.

O impacto dos programas de transferência de renda, sobretudo o PBF, na SAN também foi abordado entre os artigos publicados. Sperandio et al. ${ }^{33}$ observaram que os beneficiários do PBF das regiões Nordeste e Sudeste consumiram menos alimentos processados e ultraprocessados e também verificaram que o consumo de alimentos in natura ou minimamente processados foi superior na região Nordeste em relação à Sudes$\mathrm{te}^{33}$. Estudo transversal de base populacional observou alta prevalência de insegurança alimentar entre as famílias integrantes do $\operatorname{PBF}(81,6 \%)$. Mesmo diante desse resultado, os autores enfatizaram o papel relevante que a transferência condicionada de renda desempenha na proteção social da população ${ }^{34}$.

Outro ponto abordado é o desafio de articular os programas de transferência condicionada de renda com outros programas que promovem acesso aos direitos sociais (serviços de saúde, educação, capacitação profissional, geração de emprego e renda, saneamento, água potável e habitação)de forma intersetorial, buscando combater a insegurança alimentar, as desigualdades socioeconômicas e garantir aos mais excluídos o ingresso ao mundo dos direitos ${ }^{34,35}$.

Em relação aos grupos minoritários e SAN (quilombolas, indígenas, agricultores, assentados, entre outros), observou-se que os artigos publicados evidenciam que esses grupos apresentaram alta prevalência de insegurança alimentar e nutricional. Tais resultados sugerem que o alcance da SAN tem que perpassar pelo direito de acesso aos recursos e meios para produzir alimentos seguros e saudáveis que possibilitem uma alimentação condizente com os hábitos e práticas da região ${ }^{36}$.

Uma questão que foi escassamente discutida foi a agricultura familiar que é considerada de extrema relevância para a política de SAN, para o desenvolvimento local, para a diminuição do êxodo rural e para a comercialização da grande maioria de sua produção para o mercado local e regional $^{37}$.

Sobre Alimentação Escolar, a primeira publicação na C\&SC ocorreu em 2010 e 5\% (n = 26) do montante de artigos da área de $A \& N$ diz respeito a essa temática. A alimentação no âmbito escolar constitui um fator primordial como promotor de práticas saudáveis de $\mathrm{A} \& \mathrm{~N}$ que visa à garantia de SAN.

A revista integra esse tema simultaneamente ao avanço do Programa Nacional de Alimentação Escolar (PNAE), com a promulgação da Lei $n^{\circ}$ 11.947, de 16 de junho de 2009 e a resolução no 26, de 17 de junho de 2013, do Fundo Nacional de Desenvolvimento e Educação. O PNAE, além de estimular a melhoria dos hábitos alimentares, incentiva o comércio e a produção local de alimentos e a comercialização de alimentos provenientes da agricultura familiar, tornando-se uma estratégia fundamental de SAN e efetivação do direito humano à alimentação adequada ${ }^{38}$.

No Brasil desenvolve-se um programa de caráter universal e com impacto social vasto que em 2015 alcançou 41,5 milhões de estudantes; 
já as estratégias internacionais correspondem a projetos localizados em determinadas escolas, municípios e regiões ${ }^{39,40}$.

Os artigos publicados pela C\&SC abordaram a alimentação escolar destacando a importância do PNAE como política que apoia o desenvolvimento infantil, por meio da nutrição adequada e da melhoria das habilidades cognitivas dos estudantes. Ademais, os mesmos associaram a alimentação escolar com a alimentação orgânica, a agricultura familiar, as recomendações nutricionais, o desperdício de alimentos, a SAN, o consumo alimentar, o estado nutricional e os hábitos alimentares.

No diálogo entre os artigos que avaliaram a alimentação escolar na C\&SC e os encontrados no PubMed ( $\mathrm{n}=132$ ), observou-se que 69,5\% das publicações internacionais são oriundas de países emergentes e com economia em desenvolvimento e apenas 22,8\% são advindas da Europa ou América do Norte ${ }^{41,42}$. O Brasil liderou o quantitativo de publicações nessa temática na América do Sul, totalizando 20 manuscritos.

\section{Os artigos publicados sobre o tema} amamentação e alimentação complementar

As publicações sobre aleitamento materno tiveram início em 2008, representando 5,5\% (n = 28) do total de artigos da área de A\&N (Tabela 1) e apresentaram regularidade de frequência, com pico entre os anos de 2010 e 2018 . Neste período diversas estratégias em prol do aleitamento materno foram adotadas, dentre elas: criação da Rede Amamenta Brasil ${ }^{43}$; implantação da Estratégia Amamenta e Alimenta Brasil; instituição da Política Nacional de Atenção Integral à Saúde da Criança $(2015)^{44}$.

Os assuntos mais prevalentes nessa temática foram: o desmame e determinantes associados ao aleitamento materno $(32,1 \%)$; seguido pela temática de atenção primária à saúde, promoção, proteção e apoio ao aleitamento materno (25\%). Outros assuntos abordados incluíram: impacto do aleitamento materno na saúde do recém-nascido, hábitos de sucção, aleitamento cruzado, substitutos do leite humano, aleitamento materno versus alimentação complementar, iniciativa hospital amigo da criança, adolescência e aleitamento e prematuridade/baixo peso e aleitamento.

A busca no PubMed com o descritor breastfeeding obteve 36.898 resultados. Foi observado que as publicações tiveram como principais temáticas: promoção, proteção e apoio ao aleita- mento materno; crescimento e desenvolvimento infantil; prematuridade; desmame e seus fatores associados; saúde materna; relação entre a amamentação e desenvolvimento de doenças crônicas não transmissíveis; e composição nutricional do leite humano.

Destaca-se que a partir do ano de 2006 foi verificado uma estabilização tanto na prevalência de amamentação exclusiva em crianças menores de seis meses quanto no aleitamento materno em menores de 24 meses de idade. Fato esse que chama a atenção e que deve ser considerado um sinal de alerta, para uma possível revisão e fortalecimento das políticas e programas de promoção, proteção e apoio ao aleitamento materno, para que os indicadores de aleitamento materno retomem a tendência ascendente ${ }^{45}$.

No que se refere ao tema alimentação complementar, as publicações tiveram início em 2010 representando $1,4 \%(n=7)$ do total de artigos da área de A\&N (Tabela 1). Os temas mais prevalentes foram a introdução alimentar/alimentação saudável e a relação entre amamentação e alimentação complementar. A busca no PubMed com o descritor complementary feeding resultou em 1.517 publicações que abordavam principalmente: alimentação saudável, amamentação versus alimentação complementar e métodos de introdução alimentar.

A partir dos achados sobre este tema, ressaltase que a primeira versão do Guia Alimentar para Crianças Brasileiras Menores de 2 Anos foi publicada em 2002, sendo revisada em 2010, ano este em que as publicações na C\&SC se iniciaram. E em 2019, ano com maior número de publicações, foi lançado o novo Guia alimentar para crianças brasileiras menores de 2 anos $^{13}$.

\section{Considerações finais}

A partir da presente revisão foi possível contemplar a abrangência com a qual o campo da A\&N foi retratado na C\&SC durante seus 25 anos de existência. Observou-se um amplo leque de temas e subtemas abordados, evidenciando uma produção com compromisso e contribuição relevante com uma agenda de desenvolvimento de políticas públicas.

Apesar de não ter sido o foco inicial, já que nos seus seis primeiros anos não foram publicados artigos na área de $\mathrm{A} \& \mathrm{~N}$, este campo do conhecimento científico ganhou espaço na C\&SC a partir de 2002, com destaques para os temas de avaliação nutricional, consumo alimentar e polí- 
ticas e programas de A\&N. Os artigos, majoritariamente originais e quantitativos, e com os mais diversos grupos populacionais, constituem um repositório importante de dados e conhecimentos para profissionais e gestores da área da saúde.

A principal limitação do presente artigo se deteve na avaliação dos artigos publicados no PubMed, dado que em determinados temas, tais como avaliação nutricional, o quantitativo de artigos foi bastante expressivo dificultando a delimitação do panorama internacional sobre a questão supracitada. Destaca-se que foi realizado um esforço para avaliação dos artigos internacionais de outros temas, tais como segurança alimentar e consumo alimentar.

Ressalta-se que em função do limite de tamanho deste artigo, utilizou-se como estratégia metodológica centralizar a análise e discussão nas quatro temáticas mais frequentes. Entretanto, conforme pode ser observado na Tabela 1, dentro do escopo editorial da C\&SC, outros temas pertinentes à multidisciplinar área de $\mathrm{A} \& \mathrm{~N}$ também foram contemplados na história dos 25 anos deste periódico.

A temática mais frequente foi avaliação do estado nutricional, que é de suma importância nas definições da agenda de A\&N do SUS. Houve uma tendência a publicações voltadas à problemática da obesidade em detrimento da desnutrição, refletindo a transição nutricional no Brasil e no mundo. As publicaçõe sobre o tema consumo alimentar acompanharam a tendência da literatura internacional e focaram na qualidade da dieta, suas repercussões nas doenças crônicas, aspectos demográficos, socioeconômicos, culturais e comportamentais em grupos populacionais específicos. Entre os programas e políticas destacou-se a SAN, englobando o uso de agrotóxicos, a intersetorialidade, o impacto dos programas de transferência de renda e determinantes associados.

Alguns temas poderiam ter sido mais explorados pela C\&SC, destacando-se: deficiência de micronutrientes, agricultura familiar, PNAE, diagnósticos e denúncias sobre o recente rompimento na implementação da Política Nacional de SAN e suas articulações intersetoriais com as políticas de proteção social e combate à fome. Além disso, publicações que promovam e popularizem o Guia Alimentar Para População Brasileira podem ser um instrumento potente para aumentar a capilarização dessas informações.

\section{Colaboradores}

VM Fonseca, D Marano, FAG Vasconcellos e F Rebello participaram da concepção, desenho do método, revisão da literatura, análises e elaboração da discussão. AD Abranches e YN Villarosa do Amaral colaboraram com a revisão da literatura, análises e elaboração da discussão. VM Xavier colaborou com os gráficos e a revisão do material. 


\section{Referências}

1. Bosi MLM, Prado SD. Alimentação e Nutrição em Saúde Coletiva: constituição, contornos e estatuto científico. Cien Saude Colet 2011; 16(1):7-17.

2. Vasconcelos FAG, Batista Filho M. História do campo da Alimentação e Nutrição em Saúde Coletiva no Brasil. Cien Saude Colet 2011; 16(1):81-90.

3. Recine E, Vasconcellos AB. Políticas nacionais e o campo da Alimentação e Nutrição em Saúde Coletiva: cenário atual. Cien Saude Colet 2011;16(1):73-79.

4. Alves KPS, Jaime PC. A Política Nacional de alimentação e Nutrição e seu diálogo com a Política Nacional de Segurança alimentar e Nutricional. Cien Saude Colet 2014; 19(11):4331-4340.

5. Souza NP, Lira PIC, Fontbonne A, Pinto FCL, Cesse EÂP. A (des)nutrição e o novo padrão epidemiológico em um contexto de desenvolvimento e desigualdades. Cien Saude Colet 2017; 22(7):2257-2266.

6. Jaime PC, Delmuè DCC, Campello T, Silva DO, Santos LMP. Um olhar sobre a agenda de alimentação e nutrição nos trinta anos do Sistema Único de Saúde. Cien Saude Colet 2018; 23(6):1829-1836.

7. Vasconcelos FAG, Machado ML, Medeiros MAT de, Neves JA, Recine E, Pasquim EM. Public policies of food and nutrition in Brazil: From Lula to Temer. Rev Nutr 2019; 32:e180161.

8. Escoda MSQ. Para a crítica da transição nutricional. Cien Saude Colet 2002; 7(2):219-226.

9. Olinto MTA, Lira PIC, Marchini JS, Kac G. Formação humana, pesquisa e produção científica na subárea de avaliação "nutrição" da Coordenação de Aperfeiçoamento de Pessoal de Nível Superior, no Brasil, de 2007 a 2009. Rev Nutr 2011; 24(6):917-926.

10. Vasconcelos FAG. The scientific production of Nutrition published by the Scientific Electronic Library under the gaze of the evaluation of the Coordination for the Development of Higher Education Personnel. Rev Nutr 2017; 30(2):147-161.

11. Brasil. Ministério da Saúde (MS). Dez passos para uma alimentação saudável: guia alimentar para crianças menores de dois anos. Brasília: MS; 2013.

12. Brasil. Ministério da Saúde (MS). Guia alimentar para a população brasileira. Brasília: MS; 2014.

13. Brasil. Ministério da Saúde (MS). Guia alimentar para crianças brasileiras menores de 2 anos. Brasília: MS; 2019.

14. Batista Filho M, Souza AI, Miglioli TC, Santos MC. Anemia e obesidade: um paradoxo da transição nutricional brasileira. Cad Saúde Pública 2008; 24(Supl. 2):s247-s257.

15. Instituto Brasileiro de Geografia e Estatística (IBGE). Pesquisa nacional de saúde, 2013:ciclos de vida: Brasile grandes regiões. Rio de Janeiro: IBGE; 2015.

16. Global Burden of Disease Collaborative Network. Global Burden of Disease Study 2015(GBD 2015) Obesity and Overweight Prevalence 1980-2015. Seattle: IHME; 2017.

17. Justino LC. Prevalência de anemia em crianças nos últimos 10 anos no Brasil [monografia]. Cuiabá: Universidade Federal de Mato Grosso; 2017.

18. Minayo MCS, Gualhano L. Qualidade da produção e do consumo de alimentos: um dos maiores desafios da saúde pública contemporânea. Cien Saude Colet 2015; 20(8):1.
19. Alves MN, Muniz LC, Vieira MFA. Consumo alimentar entre crianças brasileiras de dois a cinco anos de idade: Pesquisa Nacional de Demografia e Saúde (PNDS), 2006. Cien Saude Colet 2013; 18(11):33693377.

20. Levy RB, Castro IRR, Cardoso LO, Tavares LF, Sardinha LMV, Gomes FS, Costa AWN. Consumo e comportamento alimentar entre adolescentes brasileiros: Pesquisa Nacional de Saúde do Escolar (PeNSE), 2009. Cien Saude Colet 2010; 15(Supl. 2):3085-3097.

21. Enes CC, Silva MV. Disponibilidade de energia e nutrientes nos domicílios: o contraste entre as regiões Norte e Sul do Brasil. Cien Saude Colet 2009; 14(4):1267-1276.

22. Gadenz SD, Benvegnú LA. Hábitos alimentares na prevenção de doenças cardiovasculares e fatores associados em idosos hipertensos. Cien Saude Colet 2013; 18(12):3523-3533.

23. Baião MR, Deslandes SF. Práticas alimentares na gravidez: um estudo com gestantes e puérperas de um complexo de favelas do Rio de Janeiro (RJ, Brasil). Cien Saude Colet 2010; 15(Supl. 2):3199-3206.

24. Marques ES, Cotta RMM, Botelho MIV, Franceschini S do CC, Araújo RMA. Representações sociais sobre a alimentação da nutriz. Cien Saude Colet 2011; 16(10):4267-4274.

25. Ferreira VA, Magalhães R. Práticas alimentares cotidianas de mulheres obesas moradoras da Favela da Rocinha (Rio de Janeiro, RJ, Brasil). Cien Saude Colet 2011; 16(6):2983-2991.

26. Silvani J, Buss C, Pena GG, Recchi AF, Wendland EM. Consumo alimentar de usuários do Sistema Único de Saúde segundo o tipo de assistência e participação no Bolsa Família. Cien Saude Colet 2018; 23(8):25992608.

27. Canuto R, Fanton M, Lira PIC. Iniquidades sociais no consumo alimentar no Brasil: uma revisão crítica dos inquéritos nacionais. Cien Saude Colet 2019; 24(9):3193-3212.

28. Louzada MLC, Martins APB, Canella DS, Baraldi LG, Levy RB, Claro RM, Moubarac J-C, Cannon G, Monteiro CA. Ultra-processed foods and the nutritional dietary profile in Brazil. Rev Saúde Pública 2015; 49:38.

29. Oliveira MSS, Silva-Amparo L. Food-based dietary guidelines: a comparative analysis between the Dietary Guidelines for the Brazilian Population 2006 and 2014. Public Health Nutr 2018; 21(1):210-217.

30. Burlandy L. A construção da política de segurança alimentar e nutricional no Brasil: estratégias e desafios para a promoção da intersetorialidade no âmbito federal de governo. Cien Saude Colet 2009; 14(3):851860.

31. Stoppelli IMBS, Magalhães CP. Saúde e segurança alimentar: a questão dos agrotóxicos. Cien Saude Colet 2005; 10(Supl.):91-100.

32. Faria NMX, Fassa AG, Facchini LA. Intoxicação por agrotóxicos no Brasil: os sistemas oficiais de informação e desafios para realização de estudos epidemiológicos. Cien Saude Colet 2007; 12(1):25-38. 
33. Sperandio N, Rodrigues CT, Franceschini SCC, Priore SE. Impact of the Bolsa Família Program on energy, macronutrient, and micronutrient intakes: Study of the Northeast and Southeast. Rev Nutr 2016; 29(6):833-844.

34. Monteiro F, Schmidt ST, Costa IB, Almeida CCB, Matuda NS. Bolsa Família: insegurança alimentar e nutricional de crianças menores de cinco anos. Cien Saude Colet 2014; 19(5):1347-1358.

35. Santos LMP, Pasquim EM, Santos SMC. Programas de transferência de renda no Brasil: um estudo multidimensional da implementação do Bolsa Escola, Bolsa Alimentação e Cartão Alimentação. Cien Saude Colet 2011; 16(3):1821-1834.

36. Almeida JA, Santos AS, Nascimento MAO, Oliveira JVC, Silva DG, Mendes-Netto RS. Fatores associados ao risco de insegurança alimentar e nutricional em famílias de assentamentos rurais. Cien Saude Colet 2017; 22(2):479-488.

37. Machado PMO, Schmitz BAS, González-Chica DA, Corso ACT, Vasconcelos FAG, Gabriel CG. Compra de alimentos da agricultura familiar pelo Programa Nacional de Alimentação Escolar (PNAE): estudo transversal com o universo de municípios brasileiros. Cien Saude Colet 2018; 23(12):4153-4164.

38. Siqueira RL, Cotta RMM, Ribeiro RCL, Sperandio N, Priore SE. Análise da incorporação da perspectiva do direito humano à alimentação adequada no desenho institucional do programa nacional de alimentação escolar. Cien Saude Colet 2014; 19(1):301-310.

39. Cesar JT, Valentim EA, Almeida CCB, Schieferdecker MEM, Schmidt ST. Alimentação Escolar no Brasil e Estados Unidos: uma revisão integrativa. Cien Saude Colet 2018; 23(3):991-1007.

40. Fundo Nacional de Desenvolvimento da Educação (FNDE). Dados Físicos e Financeiros do PNAE [Internet]. Disponível em: https://www.fnde.gov.br/index. php/programas/pnae/pnae-consultas/pnae-dados-fisicos-e-financeiros-do-pnae
41. Appleby LJ, Tadesse G, Wuletawu Y, Dejene NG, Grimes JET, French MD, Teklu A, Moreda B, Negussu N, Kebede B, Yard E, Gardiner I, Drake LJ. Integrated delivery of school health interventions through the school platform: Investing for the future. PLoS Negl Trop Dis 2019; 13(1):e0006449.

42. Gelli A, Daryanani R. Are School Feeding Programs in Low-Income Settings Sustainable? Insights on the Costs of School Feeding Compared with Investments in Primary Education. Food Nutr Bull 2013; 34(3):310-317.

43. Passanha A, Benicio MHD, Venancio SI, Reis MCG. Implantação da Rede Amamenta Brasil e prevalência de aleitamento materno exclusivo. Rev Saúde Pública 2013; 47(6):1141-1148.

44. Brasil. Ministério da Saúde (MS). Bases para a discussão da Política Nacional de Promoção, Proteção e Apoio ao Aleitamento Materno. Brasília: MS; 2017.

45. Boccolini CS, Boccolini PDMM, Monteiro FR, Venâncio SI, Giugliani ERJ. Breastfeeding indicators trends in Brazil for three decades. Rev Saúde Pública 2017; 51:108.

Artigo apresentado em 26/05/2020

Aprovado em 26/05/2020

Versão final apresentada em 28/05/2020 